\title{
Processo de implantação da estratégia e-SUS Atenção Básica nas UBS do Distrito Federal- DF
}

\author{
Process of implementation of the strategy e-sus basic attention in the UBS of the Federal District - \\ DF
}
Proceso de implementación de la estrategia de Atención Básica de e-SUS en el UBS del Distrito Fe- deral - DF

\author{
Geiselâne Batista da Silva Pereira ${ }^{1^{*}}$, Lucia de Medeiros Taveira ${ }^{2}$
}

Como citar esse artigo. Pereira, GBS; Taveira, LM. Processo de implantação da estratégia e-SUS Atenção Básica nas UBS do Distrito Federal- DF. Revista Pró-UniverSUS. 2020 Jul./Dez.; 11 (2): $19-26$.

\begin{abstract}
Resumo
Introdução: A estratégia e-SUS AB é uma inovação tecnológica, "remetendo a um SUS eletrônico" que trabalha com coleta de dados de usuários visando simplificar e colaborar com a organização do trabalho dos gestores e profissionais de saúde. Objetivo: Descrever sobre o processo de implantação do novo Sistema de Informação, estratégia e-SUS AB nas Unidades Básicas de Saúde (UBS), do Distrito Federal. Metodologia: Trata-se de um estudo qualitativo, exploratório descritivo, retrospectivo, constituído a partir da análise de séries temporais de dados secundários da Coordenação da Atenção Primaria/diretoria de Estratégia e Saúde da Família (COAPS/DESF), da Secretaria de Estado da Saúde (SES), do Distrito Federal, entre os anos de 2014 e 2018. Resultados: A implantação da estratégia e-SUS AB aconteceu de forma universal para todas as UBSs do Distrito Federal, por possuir um sistema próprio de prontuário eletrônico (PE) nas UBS informatizadas, "o Trakcare®". Conclusão: A adesão tardia pela SES/DF surpreendeu os pesquisadores, mas foi possível notar impactos positivos, quando se refere aos dados coletados de produção dos cuidados continuo do paciente por diferentes profissionais especializados dentro das UBSs, não há perca de informações quando coletadas corretamente e mediante ao cadastro pode-se realizar busca ativa de pacientes crônicos.
\end{abstract}

Palavras-chave: Estratégia Saúde da Família, Atenção Básica, Sistema de Informação em Saúde.

\begin{abstract}
Introduction: The e-SUS AB strategy is a technological innovation, "referring to an electronic SUS" that works with data collection from users in order to simplify and collaborate with the work organization of managers and health professionals. Objective: To describe the implementation process of the new Information System, e-SUS AB strategy in Basic Health Units (UBS), in the Federal District. Methodology: This is a qualitative, exploratory, descriptive, retrospective study, based on the analysis of time series of secondary data from the Primary Care Coordination / Directorate of Strategy and Family Health (COAPS / DESF), of the State Secretariat of Saúde (SES), from the Federal District, between 2014 and 2018. Results: The implementation of the e-SUS AB strategy took place universally for all UBSs in the Federal District, as it has its own electronic medical record system (PE) in the computerized UBS, "the Trakcare ${ }^{\circledR}$ ". Conclusion: Late adherence by SES / DF surprised the researchers, but it was possible to notice positive impacts, when referring to the data collected from the production of continuous patient care by different specialized professionals within the UBSs, there is no loss of information when collected correctly and through registration, active search for chronic patients can be carried out.
\end{abstract}

Keywords: Ostomy, Colostomy, Sexuality, Women's Health, Bodyimage.

\section{Resumen}

Introducción: La estrategia e-SUS AB es una innovación tecnológica, "refiriéndose a un SUS electrónico" que trabaja con la recolección de datos de los usuarios con el fin de simplificar y colaborar con la organización del trabajo de los gerentes y profesionales de la salud. Objetivo: Describir el proceso de implementación del nuevo Sistema de Información, estrategia e-SUS AB en Unidades Básicas de Salud (UBS), en el Distrito Federal. Metodología: Se trata de un estudio cualitativo, exploratorio, descriptivo, retrospectivo, basado en el análisis de series temporales de datos secundarios de la Coordinación de Atención Primaria / Dirección de Estrategia y Salud de la Familia (COAPS / DESF), de la Secretaría de Estado de Saúde (SES), del Distrito Federal, entre 2014 y 2018. Resultados: La implementación de la estrategia e-SUS AB se llevó a cabo de forma universal para todas las SBU del Distrito Federal, ya que cuenta con su propio sistema de historia clínica electrónica (PE). en el UBS computarizado, "el Trakcare®". Conclusión: La adherencia tardía por parte de SES / DF sorprendió a los investigadores, pero se pudo notar impactos positivos, al referirse a los datos recolectados de la producción de atención continua al paciente por diferentes profesionales especializados dentro de las UBS, no hay pérdida de información cuando se recolecta correctamente y mediante el registro se puede realizar la búsqueda activa de pacientes crónicos.

Palabras clave: Estrategia de Salud Familiar, Atención Básica, Sistema de Información de Salud.

Afiliação dos autores:

${ }^{1}$ Enfermeira. Universidade Paulista, Brasília-DF, Brasil. ORCID: https://orcid.org/0000-0002-2502-0564

${ }^{2}$ Professora Titular do Curso de Enfermagem da Universidade Paulista, Campus Brasília-DF. ORCID: https://orcid.org/0000-0001-9907-2183 


\section{Introdução}

O Sistema de Informação da Atenção Básica (SIAB), instituído em 1998, foi desenvolvido como instrumento gerencial para os Sistemas Locais de Saúde, no qual foi incorporado completamente na organização do SUS no país, usando como ferramenta na estratégia e saúde da família (ESF) como base central para coleta de dados. A disponibilização da base de dados do SIAB, faz parte das ações estratégicas da política definida pelo Ministério da Saúde (MS) com o objetivo de fornecer informações que subvencionem a tomada de decisão pelos gestores do Sistema Único de Saúde (SUS), e a instrumentalização pelas instâncias de Controle Social ${ }^{1}$.

Com o desenvolvimento e expansibilidade do SUS, tornou-se necessário a obtenção de dados mais precisos e confiáveis da população que auxilie tanto o processo de trabalho dos gestores e profissional da saúde, quanto ao armazenamento de dados junto ao Ministério da Saúde (MS). No entanto o SIAB possuía limitações em sua plataforma sendo incompatível com outros sistemas de informação utilizados pela Atenção Primaria da Saúde (APS) e para alinhar junto a conformação atual do cenário do SUS, o SIAB foi substituído pelo Sistema de Informação em Saúde para a Atenção Básica (SISAB), instituído pela Portaria GM/ MS n ${ }^{\circ} 1.412$, de 10 de julho de 2013, atualmente sendo vigente para financiamentos e adesões aos programas e estratégias da Política Nacional de Atenção Básica $(\mathrm{PNAB})^{2,3}$.

Com base na PNAB e no Programa Nacional de Melhoria do Acesso e da Qualidade (PMAQ), o Departamento de Atenção Básica (DAB) da Secretaria de Atenção à Saúde (SAS) do MS, instituiu a Estratégia e-SUS Atenção Básica (e-SUS AB), o qual está alinhada com a Política de Informação e Informática em Saúde (PNIIS) e o uso de padrões de interoperabilidade entre os sistemas de informação do SUS, atendendo os níveis Municipal, Distrital, Estadual e Federal tendo como objetivo, melhorar a qualidade da informação em saúde e de otimizar o uso dessas informações pelos gestores, profissionais de saúde e cidadãos ${ }^{4,5}$.

Aestratégiae-SUSABéumainovaçãotecnológica, "remetendo a um SUS eletrônico" que trabalha com coleta de dados de usuários visando simplificar e colaborar com a organização do trabalho dos gestores e profissionais de saúde, no intuito de qualificar a atenção prestada a população. Além de ser um instrumento que comporta ações das Políticas Nacionais de Alimentação e Nutrição (PNAN), de Saúde Bucal (PNSB) e de Práticas Integrativas e Complementares (PNPIC), também pode ser utilizada por todas as equipes que compõem a $\mathrm{AB}$, como o Núcleos de Apoio a Saúde da Família (NASF), Consultório na Rua (CnR) e Atenção Domiciliar (AD), realiza também acompanhamento dos Programa Saúde na Escola (PSE) e Academia da Saúde 3 .

Esta atuação integrada de informações permite realizar discussões de casos clínicos, possibilita o atendimento compartilhado entre profissionais, tanto nas UBSs, como nas visitas domiciliares; permitindo a construção conjunta de projetos terapêuticos de forma que amplia e qualifica as intervenções no território e na saúde de grupos populacionais. Essas ações de saúde também podem ser intersetoriais, com foco prioritário nas ações de prevenção e promoção da saúde 5 .

O e-SUS AB está disponível aos gestores das UBSs por um sistema informatizado único, composto por dois softwares: o software pra Coleta de Dados Simplificada (e-SUS AB CDS) que permite o cadastro dos usuários no atendimento de forma individual, e também o Software do Prontuário Eletrônico do Cidadão (e-SUS AB PEC), no qual realiza o cadastro dos indivíduos no território e organiza as agendas dos profissionais da $\mathrm{AB}$ com atendimentos e procedimentos prestados aos usuários. Com tudo, caberá ao gestor de cada UBS escolher entre instalar os dois softwares ou apenas um deles dependendo das características tecnológicas e da especificidade do território da população a ser adscrita ${ }^{6}$. As articulações deste processo de implementação estão interligadas pelo regime de colaboração entre o MS e unidades federativas. Desta forma, entre as várias responsabilidades do MS incluise fornecer: gratuitamente os softwares e-SUS AB; documentos orientadores para o uso, implantação e desenvolvimento do sistema; garantir a capacitação das secretarias estaduais de saúde (SES); e monitorar a implantação do e-SUS $\mathrm{AB}^{7}$.

Assim, através do regime de colaboração fica sugerido ao Estado e ao Distrito Federal a responsabilidade de informatizar as Secretarias Municipais e UBS, como fornecer estruturas físicas (equipamentos, computadores para servidores locais e consultórios, dispositivos moveis), capacitação das equipes (informática, saúde) e além de monitorar a implantação do software e-SUS $\mathrm{AB}^{7}$.

Dentro destas perspectivas, o presente estudo busca descrever o processo de implantação do e-SUS AB nas Unidades Básicas de Saúde (UBSs), do Distrito Federal (DF), entre os anos de 2014 a 2018.

\section{Materiais e Métodos}

Trata-se de um estudo descritivo e exploratório, de abordagem qualitativa, que visa descrever o processo de implantação do e-SUS AB nas Regiões Administrativas, do Distrito Federal, entre os anos de 2014 a 2018.

A pesquisa se baseou nos dados informados pela Diretoria Estratégia e Saúde da Família (DESF), que 
pertence a Coordenação da Atenção Primária a Saúde (COAPS) da Secretaria de Estado da Saúde (SES), do Distrito Federal.

Em virtude da natureza oficial dos dados, foi elaborado uma planilha com perguntas no qual respondiam os objetivos específicos do estudo, com base na necessidade da pesquisa uma solicitação foi protocolada junto a Secretaria de Estado de Saúde do Distrito Federal e a planilha para a coleta de dados foi entregue ao diretor responsável pela DESF no mês de novembro.

O estudo, em todas as fases de sua execução, obedeceu às exigências da Resolução 466/2012 da Comissão Nacional de Ética em Pesquisa (CNEP) que dispõe sobre as normas de pesquisa com seres humanos e incorpora sob a ótica do indivíduo e das coletividades, os quatro referenciais básicos da Bioética, Autonomia, Não maleficência e Beneficência.

Esta pesquisa foi submetida à análise do Comitê de Ética em Pesquisa da Universidade Paulista, tendo sida aprovada sob parecer CAAE-N ${ }^{\circ}$ 22179719.4.0000.5512. Não foi necessário a utilização do Termo de Consentimento Livre e Esclarecido para embasamento da pesquisa, pois ela foi fundamentada nos dados secundários da SES-DF.

\section{Resultados e Discussão}

O estado do Distrito Federal está localizado na região Centro-Oeste do país e possui 141 municípios. Com uma área/superfície de $5.760,783 \mathrm{~km}^{2}$ é dividido em 31 Regiões Administrativas (RAs) formada pela aérea Urbana e zona rural. As RAs de Brasília são formadas pelo Plano Piloto que engloba as Asas Sul e Norte, as regiões centrais são o Lago Sul, Lago Norte, Setor Sudoeste, Octogonal, Cruzeiro velho e Cruzeiro Novo. Um pouco mais distante das áreas centrais ficam as demais RAs (antigamente chamadas de "cidades satélites"), que são cidades de pequeno e médio portes, localizadas a uma distância variável entre de 6 e $25 \mathrm{~km}$ do Plano Piloto, que são: Gama, Taguatinga, Brazlândia, Sobradinho, Planaltina, Paranoá, Núcleo Bandeirante, Ceilândia, Guará, Samambaia, Santa Maria, São Sebastião, Recanto das Emas, Riacho Fundo I e II, Candangolândia Aguas Claras, Vicente Pires e Núcleo Bandeirante. Todas elas estão sob coordenação do Governador do Distrito Federal e da SUCAR - Secretaria de Estado de Coordenação das Administrações Regionais ${ }^{8}$.

Com uma população de 2.570 .160 habitantes registrados pelo IBGE no último censo de 2010, revelando uma densidade demográfica de 444,66 hab/ $\mathrm{km}^{2}$, porém hoje á estimativa registrada pelo IBGE é de 3.015.268 habitantes em 2019 e para coordenar as ações de saúde, foram criadas superintendências de acordo com a territorialização do $\mathrm{DF}^{8}$.

As definições abaixo estão de acordo com o Decreto $\mathrm{n}^{\mathrm{o}}$ 38.982, de 10 de abril de 2018. Região de Saúde Central: Asas Sul e Norte, Cruzeiro, Lago Norte, Varjão Região de Saúde Centro-Sul: Núcleo Bandeirante, Riacho Fundo I e II, Candangolândia, Guará, e Estrutural, Regiões de Saúde Norte: Planaltina, Sobradinho, Sobradinho II, Região de Saúde Sul: Gama e Santa Maria, Região de Saúde Leste: Paranoá, Itapoã e São Sebastião, Região de Saúde Oeste: Ceilândia e Brazlândia, Região de Saúde Sudoeste: Taguatinga, Vicente Pires, Samambaia e Recanto das Emas. No Gráfico 1 retrata o quantitativo de UBS em cada Região de Saúde o que equivale a um número de 170 UBSs $^{9}$.

Gráfico 1. Quantidade de UBS por Região Administrativa

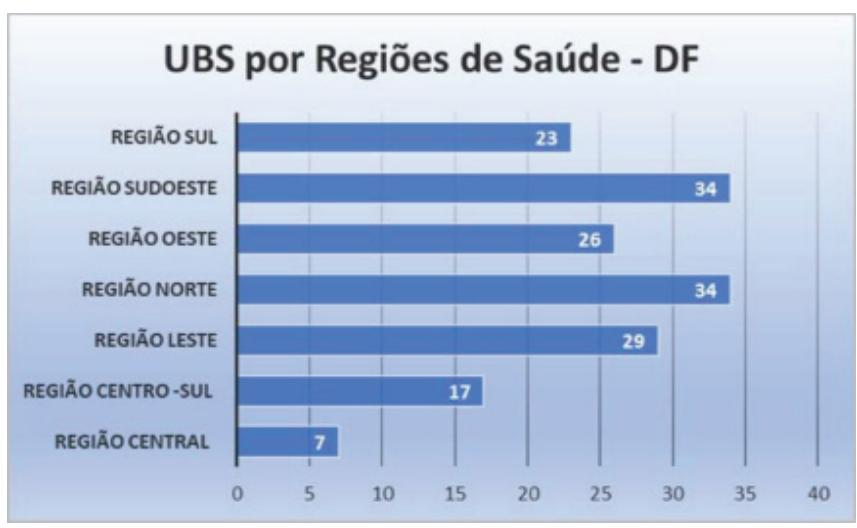

Fonte: Subsecretaria de Atenção Integral à Saúde - SAIS/site/2019

\section{Levantamento dos dados do processo de implantação}

A implantação da estratégia e-SUS AB no Distrito Federal aconteceu de forma universal para todas as equipes, porém a SES-DF retardou a implantação do e-SUS AB em 4 anos, por possuir um sistema próprio de prontuário eletrônico (PE) nas UBS informatizadas, "o Trakcare ${ }^{\circledR}$ ", e prontuários em papel para as UBS sem acesso à rede. Para garantir o envio dos dados ao SIAB a SES-DF utilizava um programa gerador disponibilizado pelo Ministério da Saúde (MS), o THRIFT/XML, um sistema para os casos de entes federados que já possuíam sistema próprio.

Vale ressaltar que a adesão ao sistema está exposta aos entes federativos de forma opcional, a depender de cada especificidade das regiões de saúde, mas sendo obrigatório o abastecimento de Dados da AB no SISAB, Assim A SES-DF refusou a implantação do e-SUS AB instituída, mediante a publicação da portaria 1.412 pelo MS em 2013, e em todas as suas prorrogações sendo a última em $2016^{5,10}$.

Apesar de algumas tentativa de se iniciar o 
processo de implantação em 2017 o mesmo só ocorreu oficialmente na SES-DF em 2018, a partir da publicação da Nota Técnica Conjunta 2/2018 - COAPS/SAIS, CTINF/SES, CCSGI/SUPLANS que versa sobre a utilização do e-SUS AB no âmbito da Atenção Primária à Saúde para registro de dados clínicos e de produção ${ }^{11}$.

A SES/DF somente aderiu ao chamado para a implantação do e-SUS AB nas UBSs do DF, devido a consolidação da Portaria GM/MS n ${ }^{\circ} 2.148$, de 28 de agosto de 2017, que estabelece o início do envio de dados de serviços da Atenção Básica (AB) para o Conjunto Mínimo de Dados (CMD) e que encerra o envio de dados do e-SUS AB para o Sistema de Informação Ambulatorial (SIA); todas as informações da atenção primaria que passaria a abastecer o CMD seria exclusivamente do SISAB, sendo este o sistema oficial de monitoramento das ações da Atenção Básica em nível nacional. A depender do cenário de implantação local, os dados poderão ser enviados de forma automática ou manual ao SISAB. sendo, o e-SUS AB o principal programa a ser utilizado para coletar e transferir para o SISAB e este para o $\mathrm{CMD}^{12}$.

O desenvolvimento do Sistema e-SUS AB, considerando todos os softwares e aplicativos, está organizado em versões. Mas para a realização da implantação da estratégia e-SUS AB, o MS disponibiliza dois softwares: Coleta de Dados Simplificada (e-SUS $\mathrm{AB} C D S$ ) e o Prontuário Eletrônico do Cidadão (e-SUS $\mathrm{AB}$ PEC), dando a opção de instalação de apenas um ou os dois softwares ${ }^{6}$. A SES-DF na estratégia de implantação optou por apenas um software, a instalação do e-SUS AB PEC.

Além disso, o MS também disponibiliza 6 cenários possíveis, de acordo com as necessidades de cada ente federativo. A SES-DF, priorizou 2 cenários que se enquadrava dentro das especificidades das UBS, conforme ilustrado nas Figuras 2 e $3^{6,13}$.

No cenário 5 a SES-DF possui atualmente $30 \%$ das 170 UBS utiliza essa versão, onde as equipes coletam todas as informações relacionada aos dados

Figura 1 . Cenário 5 para a implantação do e-SUS AB PEC.

\section{CENÁRIO 5}

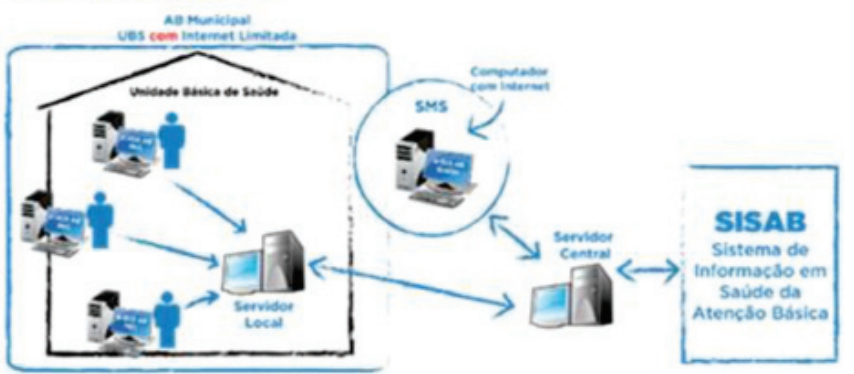

Fonte: Secretaria de Atenção Primaria a Saúde/e-SUS AB. clínicos e de produção, essas informações coletadas são guardadas no servidor local, e no período da noite é repassada ao SISAB por meio do servidor central da SES/DF. Este cenário está disponível para aquelas UBS onde a internet é limitada (internet via rádio) e não sendo possível enviar os dados online, denominado assim e-SUS AB offline ${ }^{13}$.

O cenário 6 também escolhido pela SES-DF é o chamado e-SUS AB online, (internet estável) sendo utilizado por $70 \%$ das UBS distrital, este cenário propicia ao profissional de saúde um atendimento de qualidade no qual, pode-se coletar as informações relacionada a atividade clínica e de produção e esses dados são enviados ao banco de dados do SISAB por meio do servidor central da SES-DF ${ }^{13}$.

Figura 2 . Cenário 6 para implantação do e-SUS AB PEC.

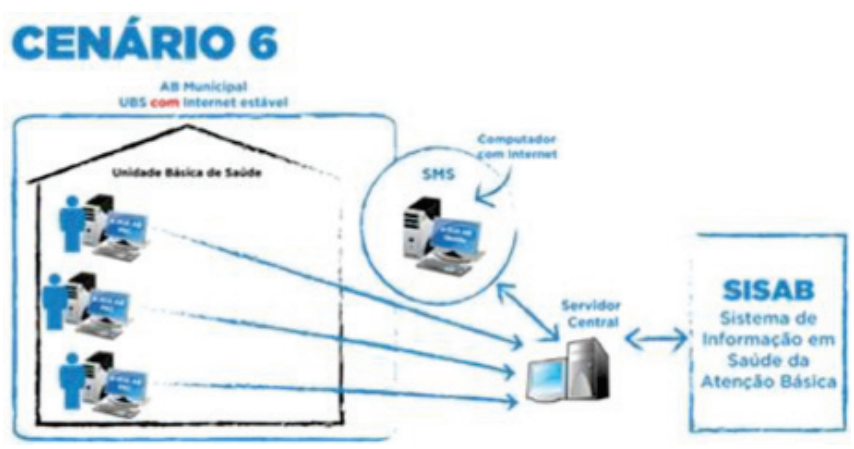

Fonte: Secretaria de Atenção Primaria a Saúde/e-SUS AB.

Mediante a necessidade de melhorias na coleta de dados pelas equipes das UBS, o MS vem atualizando as versões do PEC e a mais recente foi publicada em 2019, denominado e-SUS AB PEC v3.2 ${ }^{15}$. Estas atualizações são disponibilizadas para os entes federativos, sendo assim as UBSs do SES-DF já realizaram a atualização para o novo software.

Essa nova versão trás várias melhorias, permitindo vários registros de atividades que eram realizadas na $\mathrm{AB}$ e que os sistemas não propiciavam a coleta destas informações assim como: inclusão do módulo de avaliação do desenvolvimento da criança em atendimentos de Puericultura, inclusão da Política Nacional de Saúde Integral LGBT no cadastro do cidadão, inclusão do módulo de acompanhamento do idoso, apresentação das informações de cidadãos ativos e os que saíram do território no grupo, listagem de resultados de exames, inclusão de filtros e ordenação por data reuniões das equipes, atividades relacionadas ao Programa Saúde na Escola (PSE), funcionalidade de unificação de cadastros de cidadãos com unificação automática de prontuários ${ }^{14}$.

\section{Sobre o planejamento estratégico}

Para garantir a implantação do e-SUS AB em todo território, o MS publicou nas diretrizes nacionais de 
implantação da estratégia, um plano de Ação Regionais que pudesse auxiliar os estados, municípios e distrito federal, na capacitação de representantes das SES com apoio do DATASUS e do Departamento da Atenção Básica $(\mathrm{DAB})^{6}$.

Corroborando a essa diretriz, inicialmente os gestores do nível central da SES-DF, participaram de oficinas com a equipe técnica do MS, para entender o processo de implantação e funcionamento do sistema. A apropriação de informações especificas de uso do sistema foi realizada de forma individual, por interesse pessoal, dos técnicos das áreas de referências na SES.

$\mathrm{Na}$ sequência, as Regiões de Saúde se organizaram para realizar treinamentos em loco com o apoio da equipe técnica da SES-DF e o mapeamento das necessidades de equipamentos para funcionamento do sistema. Portanto, os técnicos do nível central da SES-DF realizaram oficinas de uso e configuração do sistema com as equipes das Gerencias de Planejamento, Monitoramento e Avaliação (GPMA), para formação de multiplicadores para treinamento dos profissionais das equipes das UBS, com apoio da equipe do nível central da SES-DF.

\section{Acompanhamento e/ou monitoramento}

Para acompanhar a efetividade da implantação do e-SUS AB, em 10 de dezembro de 2018, a SES publicou a Nota Técnica SEI-GDF n. ${ }^{0}$ 6/2018, que visa auxiliar o aprimoramento da coleta das informações durante a utilização do e-SUS AB, ela predispõe que todos os profissionais têm o dever de registrar as informações da assistência prestada ao paciente, dentro do planejamento assistencial das equipes multiprofissional de saúde das $\mathrm{UBSs}^{15}$.

Sendo assim, para o acompanhamento e monitoramento da utilização do e-SUS AB nas UBSs, a SES-DF conta com a equipe da Gerência de Processamento de Informações da Atenção Primária (GEPAP), que produz relatórios mensais sobre o envio de dados à base nacional do e-SUS AB (SISAB) bem como do quantitativo de produção.

Esses dados são encaminhados à gerência de planejamento, monitoramento e avaliação das regiões (GPMA) e para os Diretores Regionais de Atenção Primária à Saúde (DIRAPS) para acompanhamento e identificação de algum problema ou intervenção, caso seja necessária.

Pode-se notar no Gráfico 2 abaixo o número de relatórios enviados ao SISAB pela UBS do DF. Esses dados foram enviados mensalmente através da coleta

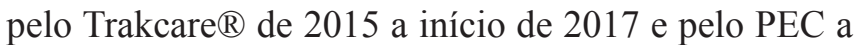
partir do final de 2017 a 2018.

É possível notar o aumento do número de relatórios produzidos pelas UBSs do Distrito Federal e
Gráfico 2. Relatórios enviados ao MS/SISAB anualmente (2014 -2018)16.

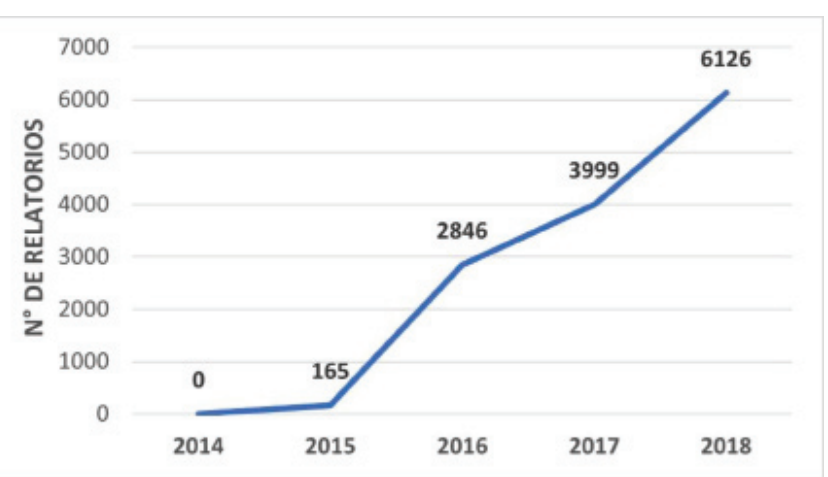

Fonte: Sistema de Informação em Saúde para a Atenção Básica/ SISAB,

enviados ao SISAB entre os anos de 2017 e 2018 após a implantação do e-SUS AB PEC.

Outro ponto importante de acompanhamento no nível central da SES-DF é a existência de uma equipe de TI responsável pela manutenção do banco de dados de todos os Sistemas de Informação utilizados e pela aquisição de insumos e equipamentos de TI e que cada região de saúde possui um Núcleo de Tecnologia da Informática (NTINF), responsável pela manutenção de equipamentos, configuração de redes e suporte aos sistemas de informações utilizados na SES-DF. Ressaltado no Decreto $\mathrm{n}^{\circ}$ 39.546, de 19 de dezembro de 2018, que traz como competência dos NTINF, quando couber; participar na elaboração e revisão de documentos normativos, prestar suporte técnico ao sistema de informações em vigência, a todas as redes de comunicação e demais ferramentas ou aplicativos que a SES-DF esteja utilizando ${ }^{17}$.

\section{Dificuldades encontradas durante a implantação}

Em estudo realizado no Mato Grosso do Sul sobre a implantação da estratégia e-SUS AB, observou-se dificuldades no processo inicial em relação a estrutura física, o que gerou em muitos municípios a implantação apenas do e-SUS CDS, pode-se perceber também que houve pouca ênfase em capacitação das equipes requerendo um acompanhamento mais próximo das equipes para o uso do sistema ${ }^{18}$.

Jápara a SESDF/DESF/COAPS, um dos primeiros desafios, foi a grande quantidade de profissionais nas UBS para capacitar no uso do sistema, aliado aos diferentes perfis dos profissionais para o registro de dados no e-SUS AB (ACS, técnicos administrativos, médicos, enfermeiros, odontólogos, técnicos de enfermagem e de higiene dental). Isso fez com que diferentes momentos de treinamento acontecem e à medida que algum 
profissional ficava mais apropriado no uso do sistema tornava-se referência para os demais profissionais para as dúvidas que surgiam. Esse movimento de troca entre os profissionais foi significativo e fundamental para o uso do sistema.

Uma outra dificuldade, foi relacionada a infraestrutura tecnológica nas UBSs, pois nem todas estão conectadas na rede interna da SES-DF, em virtude de não terem contratos de internet previstos ou por não existir cabeamento de fibra óptica na região dessas UBSs, principalmente as localizadas em área rural. Esta dificuldade foi prevista pelo MS disponibilizando assim cenários de implantação de acordo com cada especificidade local ${ }^{13}$.

A SESDF/DESF/COAPS ressalta também, que a melhoria na coleta de dados vem ocorrendo de forma gradativa e constante, mas nos meses iniciais da implantação houve uma queda de produção pela dificuldade de uso do sistema e da organização do processo pelas equipes.

Essas dificuldades iniciais podem-se notar em outros estudos como em Divinópolis/MG, onde os profissionais apresentaram dificuldades em utilizar o sistema, pela rejeição em aderir a inovação tecnológica ou capacidade técnica em manuseá-lo, os funcionários da $\mathrm{AB}$ ainda não reconheciam a estratégia e-SUS $\mathrm{AB}$ como uma ferramenta de auxílio na assistência e planejamento dos cuidados ${ }^{20}$.

\section{Impactos Relevantes na Implantação do e-SUS AB}

De acordo com o representante da SESDF/DESF/ COAPS, alguns pontos positivos se apresentaram com a implantação do e-SUS AB como descrito a seguir:

Ter um único prontuário na APS das UBSs do Distrito Federal, foi muito relevante para organizar as informações clínicas dos pacientes e essas informações estarem organizadas de forma eletrônica, ajudou no registro do atendimento e das equipes.

Para a SESDF/DESF/COAPS a organização das informações em PEC, trouxe a possibilidade de compartilhamento das informações clínicas entre diferentes equipes e diferentes UBSs o que diminui a perda de registro de dados, já que esses eram realizados em formulário de papel o que permitiu um melhor acompanhamento das condições de saúde dos pacientes.

Corroborando a este achado, pode-se perceber no estudo realizado em Belo Horizonte onde os autores cita que os profissionais anuem-se na facilidade em que o e-SUS AB tem de compartilhar as informações, gerando agilidade e rapidez no processo de atendimento, uma vez que o cadastro do paciente já se encontra a disposição dos profissionais de saúde, seja médico, ACS, enfermagem, entre outros que atuam na $\mathrm{AB}$. Esse compartilhamento das informações, também é reafirmado pelos autores como benefício, para troca de experiência, qualidade na assistência prestada, efetividade, resolutividade e eficiência dos cuidados prestados e avaliação dos casos clínicos para tomada de decisão ${ }^{21}$.

Segundo a SESDF/DESF/COAPS, o e-SUS $\mathrm{AB}$ também permitiu o registro de várias atividades que eram realizadas na APS e que os sistemas não propiciavam o registro, tal como, reuniões de equipe, atividades relacionadas ao programa saúde na escola, reuniões intersetoriais, práticas corporais, atividades de educação permanente, práticas integrativas em saúde etc. Também permitiu o registro das ações realizadas pelos Núcleos Ampliados de Saúde da Família e Atenção Básica (NASF-AB), das equipes de consultório na rua e das equipes de saúde prisional que não possuíam nenhum sistema para o registro das atividades.

Assim, se tem percebido uma grande melhora na qualidade das informações produzidas pelas equipes do DF, seja de produção direta dos profissionais (número de consultas, reuniões de equipe, visitas domiciliares, procedimentos realizados) seja de produção indireta relacionada a integração com outros sistemas como o Programa Bolsa Família (PBF) em que se tem aumentado o acompanhamento das condicionalidades relacionados ao programa a partir da implantação do e-SUS AB e do SISVAN (sistema de vigilância alimentar e nutricional).

Estas vertentes mencionadas além de estar especificada na portaria de implantação de 2013, as orientações na utilização veio se abrangendo por outras normativas como as notas técnicas publicadas diante de reuniões realizadas pelos Conselho Nacional de Secretários de Saúde (CONASS) e Conselho Nacional Secretários Municipais de Saúde (CONASEMS) que também ressalta sobre o envio de dados coletados para o SISAB, onde se afirmam que o e-SUS AB além de abastecer informações de sistemas paralelos, ele também deve ser utilizado por todas as estratégias e programas de assistência públicas da APS $^{5,6,7}$.

Outro ponto importante na implantação do e-SUS $\mathrm{AB}$ nas UBSs do DF diz respeito ao fortalecimento das ações gerenciais e de saúde no território adscrito, o que permite conhecer que territórios estão mais ou menos sobrecarregados para obter uma discussão mais qualificada da territorialização das equipes, bem como quais as áreas mais vulneráveis de cada equipe. A qualidade dos dados permite a gestão identificar fragilidades nas equipes e programar ações voltadas para os problemas encontrados.

Um estudo que também corroborou com o fortalecimento das ações gerenciais evidenciou que 
para ter uma qualidade de saúde da população da $\mathrm{AB}$, é necessário que as equipes sejam capacitadas para coletar todas as informações necessárias via e-SUS PEC, organizando e armazenando-as para se ter uma qualidade na prevenção, promoção, tratamento e reabilitação do paciente da APS, gerando impacto nos índices de saúde populacional ${ }^{20}$.

$\mathrm{Na}$ pesquisa apresentada em Sobral, um levantamento de extrema importância foi o papel gerencial na implantação do e-SUS. Ela demostrou que o envolvimento diário apoiador do gerente da UBS, foi indispensável para a capacitação e resolução de dúvidas da equipe em relação a utilização do sistema. Este apoio fez com que a equipe se empenhasse garantindo o sucesso na organização e coleta dos dados que atingiria as metas propostas pelo MS impactando no fortalecimento das ações assistenciais ${ }^{19}$.

A SESDF/DESF/COAPS entende que os dados de produção permitem que a equipe e a gestão local (gerente) organizem as ações de acordo com a relevância epidemiológica identificada e que realizem busca ativa dos pacientes que apresentam maiores riscos para determinadas condições, principalmente crianças e pacientes sob cuidados crônicos (hipertensão, diabetes e obesidade).

No artigo de Sobral-CE, os autores também evidenciaram que os profissionais além de conhecerem a estratégia lançada pelo MS em seu formato de sistema tecnológico, eles compreendem a suma importância do sistema pertinente ao perfil epidemiológico do território adscrito, levando a uma busca de planejamento de ações voltadas para assistência de pacientes crônicos ${ }^{19}$.

\section{Conclusão}

A Atenção da Saúde Primaria, no Brasil vem se tornando o foco principal dos entes federativos, por doutrinar que a porta de entrada dos usuários do SUS deve ser a Atenção Básica. Assim, para buscar um aprimoramento no atendimento e qualidade da assistência aos usuários associando-se também a possível qualidade no desempenho da realização do trabalho pelo profissionais de saúde, o MS trouxe uma das maiores ferramentas de informação, o e-SUS $\mathrm{AB}$ que visa aprimorar a coleta de dados sobre a saúde dos indivíduos nos atendimentos seja coletivo ou individual.

A adesão tardia pela SES/DF surpreendeu os pesquisadores, uma vez que ela já teria toda uma estrutura física e tecnológica, adquirida com recursos do projeto QualiSUS-Redes do MS em 2014 e 2015 e outra parte por aquisição própria da SES-DF para uso do prontuário eletrônico (Trakcare ${ }^{\circledR}$ ) nas equipes.

Assim, mesmo não aderindo ao sistema nas primeiras portarias e normativas do MS, foi possível notar impactos positivos, como continuação do atendimento do paciente por diferentes profissionais especializados dentro das UBSs, não houve perca de informações quando coletadas corretamente e mediante ao cadastro pode-se realizar busca ativa de pacientes crônicos.

Diante do exposto, nota-se que ainda há uma fragilidade quanto ao uso de evidências coletadas para desenvolvimento de pesquisas e políticas prioritárias na APS, sobretudo quanto a utilização efetiva dos dados já informatizados na saúde. Assim, espera-se que a SESDF continue a contribuir com ações planejadas dentro das UBSs, para obter resultados de informações com qualidade no atendimento individual e coletivo dos usuários da APS.

\section{Referências}

1. Ministério da Saúde. Secretaria Executiva. Datasus [acesso em abril. 2019]. Informações de Saúde. Sistema de informação da saúde. [internet] disponível em: http://www2.datasus.gov.br/SIAB/index.php?area=01

2. Ministério da Saúde. Secretaria de Atenção à Saúde. Departamento de Atenção Básica. Nota Técnica - Esclarecimentos e orientações sobre a estratégia e-SUS AB. [Acesso em: 12 de abril 2019]. Disponível em http://189.28.128.100/dab/docs/portaldab/documentos/nota_tecnica prorrogacao eSUS-AB.pdf

3. Ministério da Saúde. Secretaria de Atenção à Saúde. Departamento de Atenção Básica. Nota Técnica CONAS-Atualização sobre os sistemas de informação da Atenção Básica [online]. Brasília (DF); 2013. Disponível em: https://www.conasems.org.br/wp-content/uploads/2014/07/images NT ESUS SISAB 1.pdf

4. Ministério da Saúde. Gabinete do Ministro, Portaria No 2.073, de 31 de agosto de 2011, regulamenta o uso de padrões de interoperabilidade e informação em saúde para sistemas de informação em saúde no âmbito do Sistema Único de Saúde, nos níveis Municipal, Distrital, Estadual e Federal, e para os sistemas privados e do setor de saúde suplementar.

5. Brasil. Ministério da Saúde. Secretaria de Atenção à Saúde Departamento de Atenção Básica. Portaria n ${ }^{\circ}$ 1412, de 10 de julho de 2013. Institui o Sistema de Informação em Saúde para a Atenção Básica (SISAB). Diário Oficial da União 2013; 11 jul.

6. Brasil. Ministério da Saúde. Secretaria de Atenção à Saúde. Departamento de Atenção Básica. Diretrizes nacionais de implantação do e-SUS AB. Brasília; 2014. Modo de acesso: World Wide Web: <www.saude. gov.br/bvs $>$

7. Conselho Nacional dos Secretários de Saúde. CONASS- NOTA TÉCNICA 07 | 2013, Brasília, 19 de abril de 2013, (atualizada em julho de 2013 com a Portaria n ${ }^{\circ} 1.412$, de 10 de julho de 2013) estratégia e-sus atenção básica e sistema de informação em saúde da atenção básica - SISAB.

8. Instituto Brasileiro de Geografia e Estatística. Banco de dados IBGE Estados@. Brasília DF; 2019. [Acesso em 15 de setembro de 2019] Disponível em: https://www.ibge.gov.br/cidades-e-estados/df.html/

9. Brasil, Decreto $N^{\circ}$ 38.982, DE 10 DE ABRIL DE 2018. Altera a estrutura administrativa da Secretaria de Estado de Saúde do Distrito Federal e dá outras providências. Diário Oficial do Distrito Federal, pg. $18 \mathrm{~N}^{\circ} 69$, quarta-feira, 11 de abril de 2018

10. Ministério da Saúde (Brasil) Portaria No 1.113 , DE 31 de julho de 2015 , altera o $\S 3^{\circ}$ do art. $3^{\circ}$ da Portaria $n^{\circ} 1.412 / \mathrm{GM} / \mathrm{MS}$, de 10 de julho de 2013, que institui o Sistema de Informação em Saúde para a Atenção Básica (SISAB). [Acesso em setembro de 2019]. Disponível em: http://bvsms. saude.gov.br/bvs/saudelegis/gm/2015/prt1113_31_07_2015.html.

11. Secretaria de Estado de Saúde do Distrito Federal, Subsecretaria de 
Atenção Integral à Saúde, Coordenação de Atenção Primária à Saúde. Nota Técnica SEI-GDF n. ${ }^{\circ}$ 2/2018 - SES/SAIS/COAPS, Nota Técnica Conjunta 2018 - COAPS/SAIS/SES, CTINF/SES e CCSGI/SUPLANS/SES BrasíliaDF, 25 de junho de 2018

12. Brasil. Ministério da Saúde, Gabinete do Ministro, Portaria n $^{\circ} 2.148$, de 28 de agosto de 2017 que estabelece o início do envio de dados de serviços da Atenção Básica para o Conjunto Mínimo de Dados (CMD) e encerra o envio de dados para o Sistema de Informação Ambulatorial (SIA). [Acesso em: 28 de setembro de 2019]. Disponível em:http://bvsms.saude.gov.br/bvs/ saudelegis/gm/2017/prt2148_14_09 2017.htm

13. Secretaria de Estado de Saúde do Distrito Federal, Coordenação Primaria Atenção à Saúde, Diretoria Estratégia Saúde da Família/ SES/ COAPS/DESF. Situada STN - Asa Norte, Brasília - DF, 70086-900.

14. Brasil. Ministério da Saúde, Secretaria-Executiva. Secretaria de Atenção Primária à Saúde. e-SUS Atenção Básica, Manual de Uso do Sistema com Prontuário Eletrônico do Cidadão - PEC (versão 3.2) 2019, [Acesso em 01 de outubro 2019]. Disponível em: http://aps.saude.gov.br

15. Secretaria de Estado de Saúde do Distrito Federal, Subsecretaria de Atenção Integral à Saúde, Coordenação de Atenção Primária à Saúde. Nota Técnica SEI-GDF n. ${ }^{\circ}$ 6/2018 - SES/SAIS/COAPS Brasília-DF, 10 de outubro de 2018.

16. Brasil, Ministério da Saúde, Secretaria da Atenção Primaria a Saúde (SAPS). [acesso em 20 de novembro de 2019]. Disponível em http://aps. saude.gov.br/ape/esus

17. Brasília, Secretaria de Estado de Saúde do Distrito Federal. Decreto ${ }^{\circ}$ 39.546, de 19 de dezembro de 2018. Aprova o Regimento Interno da Secretaria de Estado de Saúde. [Acesso em 15 de outubro de 2019]. Disponível em : http:// www.sinj.df.gov.br/sinj/Norma/c7d8594440ea48969cee564fafa77865/ Decreto_39546_19_12_2018.html

18. Astolfo S, Kehrig RT. O processo de implantação de uma estratégia integrada de SIS na APS no Mato Grosso, Brasil. Rev. Saúde Col. UEFS. 2017;7(1):8-15 [acesso em 11 abril 2019]. Disponível em: http://dx.doi. org/10.13102/rscdauefs.v7i1.1169.

19. Ribeiro, Aguiar M, Muniz, Farias TB, Albuquerque, Napoleão IM`A, Vasconcelos, et al. Processo de implantação do e-SUS Atenção Básica em Sobral CE RECIIS (Online); 12(3): 1-10, jul.-set. 2018. | LILACS | ID: biblio-916722. [Acesso em 20 de outubro de 2019] Disponível em: https:// pesquisa.bvsalud.org/bvsvs/resource/pt/biblio-916722

20. Mourão AD, Neves JTR. Impactos da implantação do prontuário eletrônico do paciente sobre o trabalho dos profissionais de saúde da Prefeitura Municipal de Belo Horizonte. In: Anais do Simpósio de Excelência em Gestão e Tecnologia; 2007 Out 22-24; Resende. Rio de Janeiro. [acesso 12 outubro de 2019]. Disponível em: http:// www.aedb.br/seget/artigos2007. php?pag $=34$ 